\title{
Pseudo-SU(4) Model and Beta Decay of pf-shell Nuclei
}

\author{
J. P. Valencia \\ Instituto de Fisica, Universidad de Antioquia, Medellin, Colombia \\ and H.C. Wu \\ Instituto de Fisica, Universidad de Antioquia, Medellin, Colombia
}

Received on 26 September, 2003

\begin{abstract}
The spectra and the beta decay of the $\mathrm{Ni}-\mathrm{Cu}-\mathrm{Zn}$ isotopes with $\mathrm{A}=58,59$ and 60 are studied by using the pseudo-
\end{abstract} SU(4) model.

\section{Introduction}

The pf-shell (i.e. the $2 \mathrm{p}_{\frac{1}{2}}-2 \mathrm{p}_{\frac{3}{2}}-1 \mathrm{f}_{\frac{5}{2}}$ shell) is important for a variety of problems in nuclear structure, however, the study of this shell has been hindered by the huge size of the shell model space. In recent years there have been studies by using the Monte-Carlo shell model [1] and that based on the pseudo-SU(4) symmetry model [2].

The original Wigner Supermultiplet SU(4) model[3] is based on the spin-isospin symmetry of the nucleon-nucleon interaction. But the symmetry is broken by the l-s coupling and the breaking increases with the nucleon number. It has been established that the SU(4) symmetry works well in the p-shell $[4,5]$, but for the ds-shell the SU(4) symmetry is good only for nuclei with $N \cong Z[6,7]$. For nuclei beyond ds-shell the SU(4) symmetry is heavily broken due to large 1-s coupling.

There have been efforts to restore symmetry for the nuclei which have large 1-s coupling by introducing the concept of pseudo-spin and pseudo-orbit[8]. The pseudo-SU(4) model [2] was proposed to deal with the pf-shell by combining the pseudo-spin and the isospin. Since the pseudospin-pseudo-orbit splitting (between $1 \mathrm{f}_{\frac{5}{2}}$ and $2 \mathrm{p}_{\frac{3}{2}}$ ) is substantially smaller than the normal spin-orbit splitting (i.e. between $2 \mathrm{p}_{\frac{1}{2}}$ and $2 \mathrm{p}_{\frac{3}{2}}$ ), the breaking of the pseudo-SU(4) symmetry is correspondingly smaller. The advantage of the pseudo-SU(4) symmetry is that it keeps all the algebraic features of the SU(4) model while incorporating large 1-s coupling. A brief analysis in ref.[2] indicates that the pseudoSU(4) model is promising for describing the pf-shell nuclei.

This work intends to check the validity of the pseudoSU(4) model by a comprehensive analysis of the spectra and the beta decays of the Ni-Cu-Zn isotopes with $A=58,59$ and 60 .

\section{The Spectra}

In the pseudo-SU(4) model we further assume that the pseudo-orbits have the SU(3) symmetry [5]. Note that here- after the symmetries pseudo-SU(4) and pseudo-SU(3) are denoted as SU(4) and SU(3), respectively. Therefore the symmetry group chain for the pf-shell nuclei reads,

$\mathrm{SU}(4) \otimes \mathrm{SU}(6) \supset\left(\mathrm{SU}(2)_{\mathrm{S}} \otimes \mathrm{SU}(2)_{\mathrm{T}}\right) \otimes(\mathrm{SU}(3) \supset \mathrm{SO}(3))$,

where the SU(4) and SU(6) are the pseudo-spin-isospin symmetry and pseudo-orbital symmetry, respectively; $\mathrm{SU}(2)_{\mathrm{S}}$ and $\mathrm{SU}(2)_{\mathrm{T}}$ and $\mathrm{SU}(3)$ are their subgroups. A general form of the energy can be expressed as,

$$
\begin{aligned}
E= & a_{1} C_{\mathrm{SU} 4}+a_{2} S(S+1)+a_{3} T(T+1)+a_{4} C_{\mathrm{SU} 3} \\
& +a_{5} L(L+1)+a_{6}\langle L \cdot S\rangle,
\end{aligned}
$$

where $C_{\mathrm{SUN}}$ are the second order Casimir operator of the $\mathrm{SU}(\mathrm{N})$ group, and $S, T, L$ and $J$ are the spin, isospin, the orbital angular momentum and the total angular momentum, respectively.

First we study the Ni-Cu-Zn nuclei with $A=58$, or $n=2$ ( $n$ being the number of nucleons in the pf-shell). The irrep of $\mathrm{SU}(4) \otimes \mathrm{SU}(6)$ is as follows:

where $\square$ is the SU(4) irrep, and it can be decomposed to the states with $(S T)=(01)$ and (10); $\square$ the $S U(6)$ irrep and its SU(3) irrep with largest symmetry is $(4,0)$. The left hand side of Figure 1 lists the experimental levels with lowest energies. We note that the experimental energy given in Figure 1 is the pure nuclear interaction, i.e. the binding energy with Coulomb energy being subtracted, and the nuclear energy of the ground state of ${ }^{58} \mathrm{Zn}$ is taken as zero. The quantum numbers $S$ and $L$ are also given to experimental levels in order to identify their theoretical counterparts.

Since all the levels belong to the same SU(4) irrep, and the $(S T)=(01)$ and $(10)$, only two parameters from eq. 2 are needed, which are $a_{3}$ and $a_{5}$. From fitting the five levels the two parameters can be determined as : $a_{3}=0.114$ and $a_{5}=$ 0.200 (all the parameters $a_{i}$ are in $\mathrm{MeV}$ ). The calculated spectra are as given in the right hand side of Fig. 1. We note that only the levels with lowest energies are listed. 


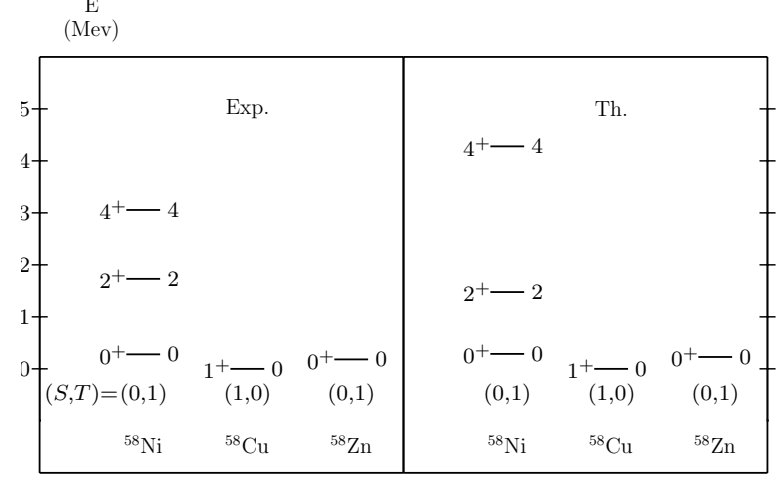

Figure 1. The spectra of the $\mathrm{Ni}-\mathrm{Cu}-\mathrm{Zn}$ nuclei with $\mathrm{A}=58$. The experimental energies are pure nuclear interactions, and the theoretical levels given by the $\mathrm{SU}(4) \otimes \mathrm{SU}(6)$ model. For each level $J^{\pi}$ (to the left) represents the total angular momentum and parity, and $L$ (to the right) the orbital angular momentum. The spin-isospin number, $(S, T)$, are given for each nucleus.

Considering that the energy difference between the ground states of ${ }^{58} \mathrm{Ni}$ and ${ }^{58} \mathrm{Zn}$ is less than $100 \mathrm{KeV}$ while the total nuclear energies are around $634 \mathrm{MeV}$, one can easily conclude that the $\mathrm{SU}(2)_{\mathrm{T}}$ symmetry is almost exact. However, the calculated energy of $4^{+}(4.277 \mathrm{MeV})$ of ${ }^{58} \mathrm{Ni}$ is much higher than its experimental counterpart (3.053 $\mathrm{MeV})$, which shows that the $\mathrm{SU}(3)$ symmetry is violated considerably.

Next we discuss the Ni-Cu-Zn nuclei with $A=59$, or $n=3$. The irrep of $\mathrm{SU}(4) \otimes \mathrm{SU}(6)$ is:

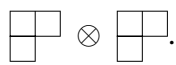

The first diagram $\square$ is the SU(4) irrep, and it can be decomposed to $(S, T)=(1 / 2,1 / 2),(1 / 2,3 / 2)$ and $(3 / 2,1 / 2)$; the second diagram $\square$ is the $\mathrm{SU}(6)$ irrep and its $\mathrm{SU}(3)$ irrep with largest symmetry is $(4,1)$.

The left hand side of Fig. 2 lists the lowest experimental levels. The calculated spectra are given in the right hand side of the same figure. The three relevant parameters can be determined through fitting the 10 levels: $a_{3}=2.014, a_{5}=$ 0.320 and $a_{6}=-0.360$. The $\mathrm{SU}(2)_{\mathrm{T}}$ symmetry is, again, almost exact for the case of $n=3$. The spectra of the three nuclei are similar and the general fair agreement between experiment and theory is an evidence of the validity of the SU(4) symmetry. We note that the angular momentum assigned to the two excited states of ${ }^{59} \mathrm{Zn}(E=0.540$ and 0.900 $\mathrm{MeV}$ ) is only tentative, and a confirmation of this assignment could provide a strong support for the SU(4) symmetry. In the experimental spectra of ${ }^{59} \mathrm{Ni}$ the $5 / 2^{-}$level is lower than $1 / 2^{-}$, which indicates that the experimental $L=2$ state is considerably lower than that predicted by the SU(3) symmetry. This is an indication of violation of the SU(3) symmetry.

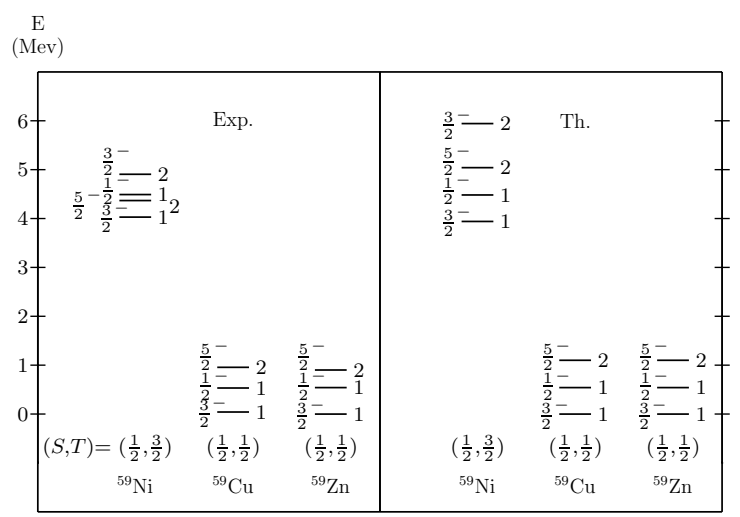

Figure 2. The spectra of the Ni-Cu-Zn nuclei with $\mathrm{A}=59$. See the caption of Fig. 1 for explanation of the signs.

Finally we discuss the Ni-Cu-Zn nuclei with $A=60$, or $n=4$. There are three sets of irreps of $\mathrm{SU}(4) \otimes \mathrm{SU}(6)$ :

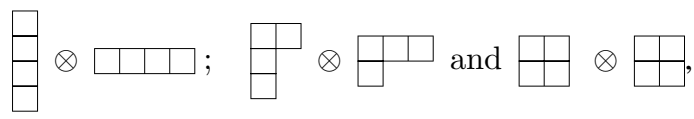

which correspond to the $\mathrm{Zn}, \mathrm{Cu}$ and $\mathrm{Ni}$ nuclei, respectively. In the first set the SU(4) irrep $\square$ can be decomposed to $(S, T)=(0,0) ; \square \square \square \square$ is the the $\mathrm{SU}(6)$ irrep and its $\mathrm{SU}(3)$ irrep with the largest symmetry is $(8,0)$. In the second set is the $\mathrm{SU}(4)$ irrep, and it can be decomposed to $(S, T)=(1,1)$, $(1,0)$ and $(0,1)$; the $\square \square \square$ is the SU(6) irrep and its $\mathrm{SU}(3)$ irrep with the largest symmetry is $(6,1)$. In the third set the SU(4) irrep $\square$ contains $(S, T)=(0,2)$ and $(1,2)$ etc, and the SU(6) irrep has the SU(3) irrep $(4,2)$ etc.

The left hand side of Fig. 3 lists the lowest experimental levels, and the calculated spectra are given in the right hand side of the figure. The four relevant parameters can be determined from fitting the eleven levels, and the results are: $a_{1}=0.550, a_{3}=0.100, a_{5}=0.167$ and $a_{6}=-0.031$.

The nuclei ${ }^{60} \mathrm{Ni}$ and ${ }^{60} \mathrm{Zn}$ have $S=0$ thus there is $J=L$. The low-lying states of ${ }^{60} \mathrm{Ni}$ are contained in the SU(3) irrep $(4,2)$ and those of ${ }^{60} \mathrm{Zn}$ are in the SU(3) irrep $(8,0)$. In both cases the levels are explained qualitatively by the SU(3) model. However, the quantitative rule of the energy of the $\mathrm{SU}(3)$ limit is violated, and the extent of the violation of SU(3) symmetry is similar to the case of $n=2$ and 3 . The ground state of ${ }^{60} \mathrm{Cu}$ is $2^{+}$which belongs to the triplet 2 , 1 , and 0 that are produced by the coupling between $S=1$ and $L=1$. Experimentally there have been identified only $2^{+}$and $1^{+}$states. Since in the spectra of $\mathrm{Cu}$ there are plenty of unidentified states, with the advance of experiment, it is quite possible that one of such undetermined states can be assigned as the missing $0^{+}$. 


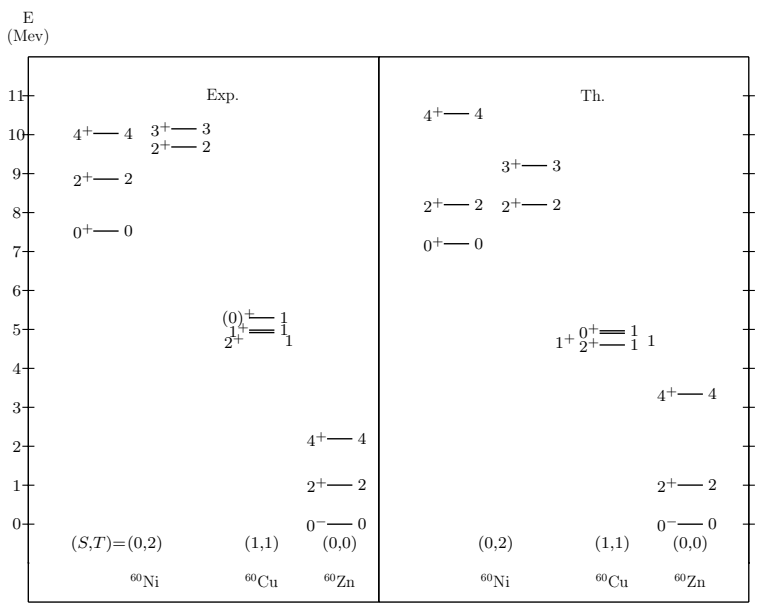

Figure 3. The spectra of the Ni-Cu-Zn nuclei with $A=60$. See caption of Fig. 1 for explanation of the signs.

\section{The beta decays}

The Gamow-Teller beta decay operator writes as

$$
M_{\mathrm{GT}}=\frac{1}{\sqrt{2 J_{i}+1}} \sum_{f, i}\left\langle f\left|\sum_{k} \tau_{k} \sigma_{k, \mu}\right| i\right\rangle,
$$

where $|i\rangle$ and $|f\rangle$ are the initial and final states with the pseudo-SU(4) symmetry, and $k$ is the particle index. For the convenience of calculation one may take $|i\rangle$ and $|f\rangle$ as the normal SU(4) state in the ds shell, provided the GamowTeller operator undergoes a transformation as follows [2],

$$
\overrightarrow{\tau_{k}} \overrightarrow{\sigma_{k}}=-\left(\frac{\overrightarrow{\tau_{k}} \overrightarrow{\sigma_{k}}}{3}+2 \sqrt{10} \overrightarrow{\tau_{k}}\left[C_{k}^{(2)} \otimes \overrightarrow{\sigma_{k}}\right]^{(1)}\right)
$$

where the quadrupole operator $C_{k}^{(2)}$ is given in ref. [10]. With the assumption that the pseudo-orbits obey the SU(3) symmetry [9], the Gamow-Teller matrix elements can be derived as follows,

$$
\begin{aligned}
M_{\mathrm{GT}}= & \sqrt{\frac{2 J_{f}+1}{\left(2 J_{i}+1\right)\left(2 T^{\prime}+1\right)}}\left\langle T M_{T} 1 \nu \mid T^{\prime} M_{T^{\prime}}\right\rangle\left\langle S^{\prime} T^{\prime}\|\sigma \tau\| S T\right\rangle \\
& \left(\frac{(-1)^{J_{f}+S+L}}{3}\left\{\begin{array}{ccc}
J_{i} & J_{f} & 1 \\
S^{\prime} & S & L
\end{array}\right\} \delta_{L^{\prime} L}-2 \sqrt{\frac{10}{3}}\left\{\begin{array}{ccc}
L^{\prime} & L & 2 \\
S^{\prime} & S & 1 \\
J_{f} & J_{i} & 1
\end{array}\right\}\left\langle L^{\prime}\left\|C^{(2)}\right\| L\right\rangle\right),
\end{aligned}
$$

where the reduced SU(4) matrix elements of $\sigma \tau$ and the reduced $\mathrm{SU}(3)$ matrix element of $C^{(2)}$ are

$$
\left\langle S^{\prime} T^{\prime}\|\sigma \tau\| S T\right\rangle=2 \sqrt{\mathrm{C}_{\mathrm{SU} 4}[\mathrm{f}]}\left\langle[f] w \phi S T ;[211] 11 \|[f] w^{\prime} \phi^{\prime} S^{\prime} T^{\prime}\right\rangle,
$$

and

$$
\begin{gathered}
\left\langle L^{\prime}\left\|C^{(2)}\right\| L\right\rangle=\sum_{\substack{(\lambda \mu), K L l l^{\prime}}}(-1)^{l-L+L^{\prime}} \sqrt{\left(2 L^{\prime}+1\right)\left(2 l^{\prime}+1\right)(2 L+1)}\left\{\begin{array}{ccc}
L & l & L \\
l^{\prime} & L^{\prime} & 2
\end{array}\right\} \\
\left\langle l^{\prime} 020 \mid l 0\right\rangle\left\langle\left(\lambda^{\prime} \mu^{\prime}\right) K^{\prime} L^{\prime} \|(\lambda \mu) K L ;(20) l^{\prime}\right\rangle\langle(\lambda \mu) K L ;(20) l \|(\lambda \mu) K L\rangle,
\end{gathered}
$$

respectively.

Figure 4 gives a comparison between calculations and experiments of the beta decay for the $\mathrm{Ni}-\mathrm{Cu}-\mathrm{Zn}$ isotopes with $A=58(n=2)$ at the upper part, and that of $A=59(n=3)$ at the lower part, respectively. In this figure, for each beta transition the $\log f t$ value, energy and $J^{\pi}$ of the final state are listed.

The comparison shows that the pseudo-SU(4) model gives predictions very close to their experimental counterparts. This is a clear indication that the pseudo-SU(4) model is good for the lowest states of these nuclei.

However, for the case of $A=60$, i.e. $n=4$, the theoretical prediction of the beta decay rate is zero due to that the initial state and the final state belong to different SU(4) irreps. This zero-beta-decay-rate prediction contradicts experiments, and it suggests that a mixing between different
SU(4) irreps is needed.

\section{Conclusions and Discussion}

This work studies the spectra and the beta decay of the lowest states for the Ni-Cu-Zn isotopes with $\mathrm{A}=58,59$ and 40. In the spectra, the lowest levels of three nuclei (with the same nucleon number A) are described by a single formula with 2, 3 or 4 parameters. It has been shown that the pseudoSU(4) is a reasonably good symmetry for the nuclei at the beginning of the pf-shell. However, for the pseudo-orbital motion the SU(3) symmetry is broken considerably. Therefore, it is of great interest to explore other types of symmetry for the pseudo-orbits. For example the $\mathrm{SU}(6) \supset \mathrm{SO}(6)$ [9] is one of such possibilities. 


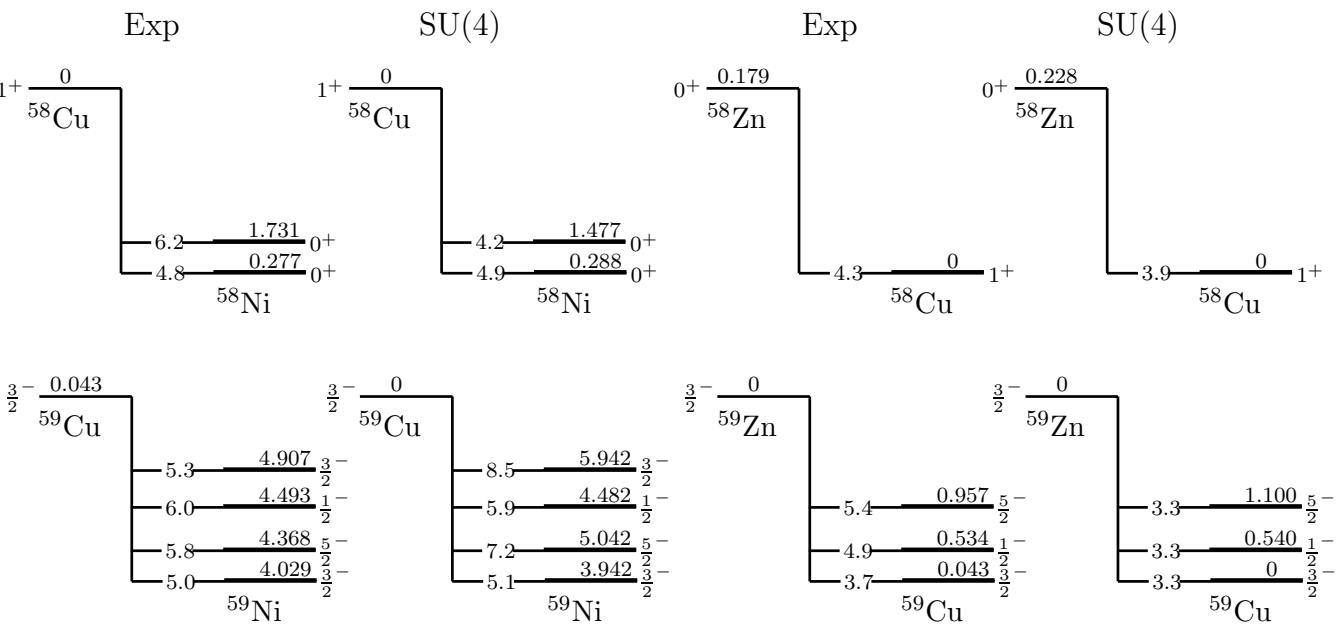

Figure 4. The beta decay of $\mathrm{Ni}-\mathrm{Cu}-\mathrm{Zn}$ isotopes with $\mathrm{A}=58$ and $\mathrm{A}=59$.

The predictions of the pseudo-SU(4) symmetry model on beta decay of the $\mathrm{Ni}-\mathrm{Cu}-\mathrm{Zn}$ isotopes with $\mathrm{A}=58$ and 59 are also in a reasonable agreement with experiments. However for $A=60$, a mixing between different $S U(4)$ irreps is needed. This discrepancy for $n=4$ also hints that the validity of the pseudo-SU(4) may decrease with the increase of $n$, which is quite similar to the situation that the original SU(4) model in the ds-shell is good at the beginning of the shell, but the goodness decreases when the number of nucleons increases [6, 7].

\section{Acknowledgments}

The authors thank the Colciencias and the University of Antioquia, Colombia, for financial support.

\section{References}

[1] M. Honma, T. Otsuka, B.A. Brown, and T. Mizusaki, Phys. Rev. C65, 061301 (2002).
[2] P. Van Isacker, O. Juliet, and F. Nowacki, Phys. Rev. Lett. 82, 2060 (1999).

[3] E.P. Wigner, Phys. Rev. 51, 106 (1937).

[4] J.C. Parikh, Group Symmetries in Nuclear Structure, Plenum Press, 1978.

[5] M. Harvey, Advances in Nuclear Physics, 1, 67 (1968)

[6] P. Van Isacker, D.D. Warner, and D.S. Brenner, Phys. Rev. Lett. 74, 4607 (1995).

[7] P. Vogel and W.E. Ormand, Phys. Rev. C47, 623 (1993).

[8] A. Arima, M. Harvey and K. Shimizu, Phys. Lett. B30, 67 (1969).

[9] P.J. Elloitt, Proc. Roy. Soc. London A245, 128 (1958).

[10] A.R. Edmonds, Angular Momentum in Quantum mechanics, Princeton University Press, 1974. 